\title{
HPV vaccines: where are we now?
}

\author{
Margaret Stanley
}

\section{Introduction}

Human papillomaviruses (HPV) are a large family of small double-stranded DNA viruses that infect squamous epithelia (or cells with the potential for squamous maturation) including the skin and the mucosae of the anogenital tract and upper respiratory tract. More than 100 HPV s have been isolated from clinical biopsies. HPVs are classified by DNA sequence and numbered in the sequence in which they were isolated (e.g. HPV 1, HPV 2, etc.). Some 30-40 HPVs regularly or sporadically infect the genital tract and here they fall into two groups: low-risk viruses such as HPV 6 and 11 that cause genital warts and high-risk viruses associated with anogenital cancer. Infection with one of a subset of 15 high-risk HPVs is the main cause of invasive cervical cancer, but two types HPV 16 and HPV 18 - cause more than $70 \%$ of carcinoma cervix, with HPV 16 detected in more than 50\% and HPV 18 in $7-20 \%$ of cases (irrespective of the geographical location). ${ }^{1}$ Although cervix cancer is the major consequence of oncogenic HPV infection, a proportion of cases of carcinoma of the penis, vulva, vagina, anus and oropharynx are attributed to HPV, with HPV 16 being the major player. Overall, it is estimated that HPV is the cause of $3.7 \%$ of all cancers, making this a seriously important human carcinogen. ${ }^{1}$

\section{Efficacy of HPV vaccines in randomised control trials}

Prophylactic vaccines designed to prevent and/or control some genital HPV infections have been developed. The vaccines are subunit vaccines consisting of virus-like particles (VLPs) made of only one protein: the major HPV coat or capsid protein, L1. HPV VLPs are made using sophisticated recombinant technology in which the L1 gene is expressed in recombinant yeast or baculovirus vectors. The chemistry of the expressed protein is such that it spontaneously assembles into VLPs that are morphologically (and more importantly) immunologically identical to the native virus but lack DNA and are therefore non-infectious. Two HPV prophylactic vaccines have been developed, these are Cervarix ${ }^{\circledR}$, a bivalent HPV 16/18 VLP vaccine from GlaxoSmithKline, and Gardasil ${ }^{\circledR}$ (also known as Silgard ${ }^{\circledR}$ ), a quadrivalent HPV 16/18/6/11 vaccine from Merck; profiles of these vaccines are shown in Table 1.

The results of Phase III double-blind, placebo-controlled, randomised trials of both vaccines have been published recently. The quadrivalent vaccine has shown, in the per protocol efficacy group (i.e. women aged 16-26 years with five or fewer lifetime sex partners, who were HPV 6, 11, 16 and 18 negative at entry), $100 \%$ efficacy against vulval intraepithelial neoplasia, vaginal intraepithelial neoplasia, genital warts and $98 \%$ efficacy against cervical intraepithelial neoplasia (CIN) 3 and adenocarcinoma in situ caused by any of the vaccine HPV types after a 3-year

J Fam Plann Reprod Health Care 2007; 33(4): 227-229

Department of Pathology, University of Cambridge, Cambridge, UK

Margaret Stanley, PhD, Professor of Epithelial Biology

Correspondence to: Professor Margaret Stanley, Department of Pathology, University of Cambridge, Tennis Court Road, Cambridge CB2 1QP, UK. E-mail: mas@mole.bio.cam.ac.uk
Table 1 Vaccine profiles

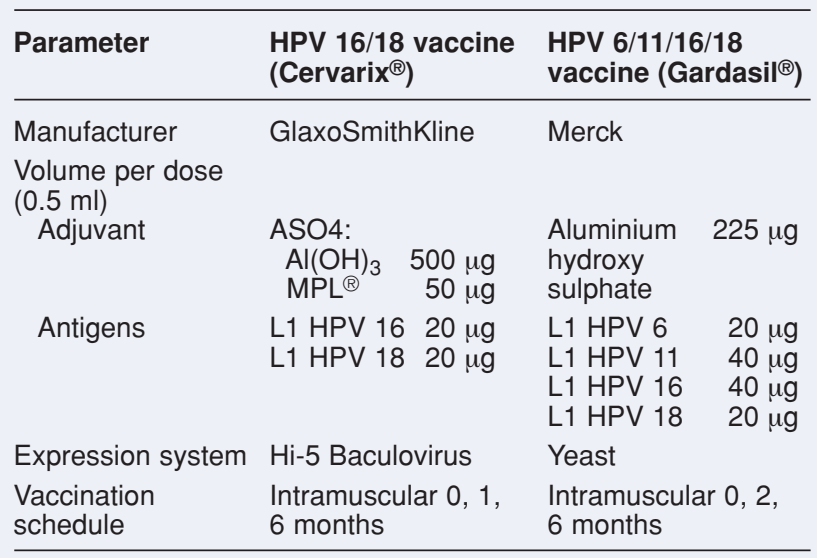

follow-up..$^{2,3}$ In an interim analysis with a mean follow-up of 14.8 months, the bivalent vaccine has shown (in women aged $15-25$ years with six or fewer lifetime sex partners and DNA negative for the relevant oncogenic HPV type in the vaccine), $90.4 \%$ efficacy against HPV 16/18 CIN 2+ (two cases in the vaccine group, 21 cases in the placebo group). ${ }^{4}$ It is unlikely that the HPV 16 or 18 infection detected in the two cases in the vaccine group caused the CIN 2+. HPV 16 or 18 DNA was not detected in any of the preceding cervical cytology samples, nor was there evidence of HPV 16 or 18 gene expression in the biopsy tissue, but another nonvaccine oncogenic HPV type was present in all sections of the biopsy and in the preceding cytology samples, including that taken at Day 0. The one case of CIN 3 in the vaccine cohort in the quadrivalent trial exhibits a similar profile. HPV 16 was detected only in the biopsy, but another nonvaccine oncogenic HPV type was present in the cytology samples at Day 0 and in every section of the large loop excision biopsy. 5

\section{Duration of protection}

Although HPV L1 VLP vaccines have shown remarkable efficacy against HPV-related disease in women aged 15-26 years and naïve for the vaccine HPV types, a key issue is how long this protection will last. At present there are no immune correlates of protection, since seroconversion has occurred in $98 \%$ or more vaccinees and there have been no obvious breakthroughs of disease in the vaccinated cohorts in both Phase III and Phase II trials. The follow-up in the latter cohort, for both vaccines, currently extends to more than 5 years. Both VLP vaccines result in high levels of serum-neutralising anti-HPV L1 immunoglobulin G ( $\mathrm{IgG})$ that at peak concentrations are up to 1000 times, and at 5-6 years up to 10-12 times, that measured in natural genital HPV infections. Mathematical modelling of the kinetics of antibody decay indicates that antibody could persist for 30 years. ${ }^{6}$ Importantly, there is good evidence that robust immune memory is generated by these vaccines. The quadrivalent vaccine has shown an impressive recall response to antigen challenge (the functional readout for memory) 5 years post-immunisation. ${ }^{7}$ Also, circulating memory B cells can be detected 1 month after the third and final immunisation with the bivalent vaccine. ${ }^{8}$ Immune memory is fundamental to successful immunisation, and the observations of persistence of antibody and robust recall from the VLP vaccine trials leads to optimism that 
the duration of protection might be measured in decades. However, as is usually the case at the time of introduction of a vaccine, the duration of protection cannot be predicted with certainty and post-vaccine surveillance and monitoring are essential.

\section{Cross-protection}

The humoral immunity induced appears to be predominantly type-specific and this raises the question of whether there is any cross-protection afforded by the vaccines since there is considerable amino acid sequence homology in L1 between closely related HPV types. There is evidence that the bivalent vaccine partially protects against persistent infection with HPV 31 and HPV $45 .{ }^{4}$ The possibility of cross-protection from the VLP vaccines is strengthened by the evidence that cross-neutralising antibodies against HPV $31 / 45 / 52 / 58$ are generated after vaccination with the quadrivalent vaccine (Smith JV, personal communication, 2006) albeit at concentrations 1-2 logs lower than the dominant type-specific neutralising antibodies. It must be emphasised, however, that at the present time there is no evidence for cross-protection against HPV 45- or 31-induced CIN 2/3, and if such cross-protection does occur then it is likely to be partial and not complete. This does imply that second-generation vaccines will need to be polyvalent vaccines and include other HPV types.

\section{Who and when to vaccinate}

Both vaccines have a gender-neutral license in the European Union for 9-26-year-olds (Gardasil) and 10-25year-olds (Cervarix). Many countries have issued recommendations as to the cohorts to be vaccinated. Most have opted for peripubertal females with varying age cohorts (or none) as a catch-up group. Only Australia has recommended immunisation of boys in the 9-15 years age group; there are no efficacy data for men at present. Recently, the UK Joint Committee on Vaccination and Immunisation issued an interim recommendation that the HPV VLP vaccine be offered to 12-13-year-olds via a school-based immunisation delivery programme. Such a recommendation makes sense from several perspectives: immune response, public health criteria and costeffectiveness. In the immunobridging studies the optimal immune response in terms of peak antibody concentration (and speculatively immune memory) was achieved in 9-12-year-olds; antibody responses declined slowly over the subsequent decade. ${ }^{9}$

The vast majority of genital HPV infections are sexually transmitted. Therefore, for effective prophylaxis, vaccination should occur before most of the population is exposed (i.e. before the onset of sexual activity). Vaccines are public health interventions, not medicines, and their efficacy in preventing disease and hence their costeffectiveness depends upon a wide coverage of the unexposed population. This objective is more likely to be achieved with a school-based programme for peripubertal females, provided it is accompanied by effective education and information regarding HPV for both the medical community and the general public. Delaying vaccination to mid- or late-adolescence is unlikely to provide the coverage necessary for cost effective immunisation, since this group participate poorly in vaccination programmes.

One question that is frequently raised is: "Why stop at 26-year-olds? Why not vaccinate, off label, older women who may be at risk?" The vaccines after all have a very good safety profile and have shown high efficacy in the 15-26-year-old group. There are no data as yet on vaccine efficacy in women aged $>26$ years and an effective secondary intervention is available for women in the form

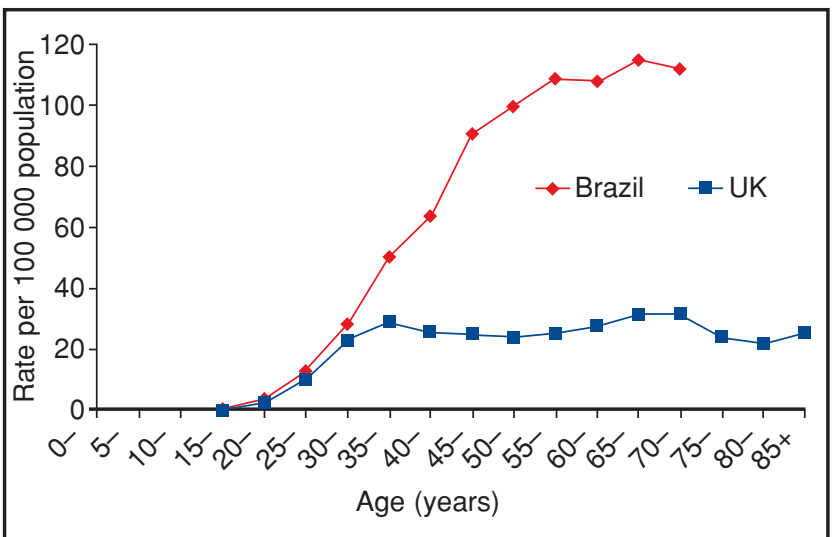

Figure 1 Age-specific incidence rates of cervical cancer in Brazil and the UK ${ }^{10}$

of the cervical cancer screening programme. Screening starts to impact on cervical cancer incidence in women aged over 30 years (Figure 1) ${ }^{10}$ but appears to have little effect on the under-30s. ${ }^{11}$ The UK has one of the most effective cervical cancer screening programmes in the world, achieving a $70 \%$ reduction in cervical cancer incidence overall. ${ }^{12}$ Since the screening programme (in one form or another) will have to continue for the foreseeable future, vaccination of 'older' women is unlikely to be costeffective in a public health programme, compared to the younger age group. It must also be remembered that the current vaccines only include two of the 15 oncogenic HPV types and, even if delivered optimally with $100 \%$ coverage, would prevent only $70 \%$ of cervical cancers in the long term. It is critical, therefore, that women do not perceive the vaccine as a 'magic bullet' giving complete protection against cervical cancer. They must continue in the screening programme, vaccinated or not. Screening methods may change in the medium term, but until a vaccine is available that prevents $90 \%$ or more of invasive cervical cancers, the need for the screening programme will continue.

So what might one expect if a magic wand was waved and all 9-26-year-old women were vaccinated tomorrow? This scenario is depicted in Figure 2 and is based on the incidence of HPV 16/18 in cytologically normal and abnormal women. ${ }^{13}$ The impact in the vaccinated cohort would be seen initially as a reduction in borderline [atypical squamous cells of undetermined significance/atypical glandular cells of undetermined significance (ASCUS/AGUS)] smears, then a reduction in CIN 1 and within 2-15 years a 50\% reduction in CIN 2/3

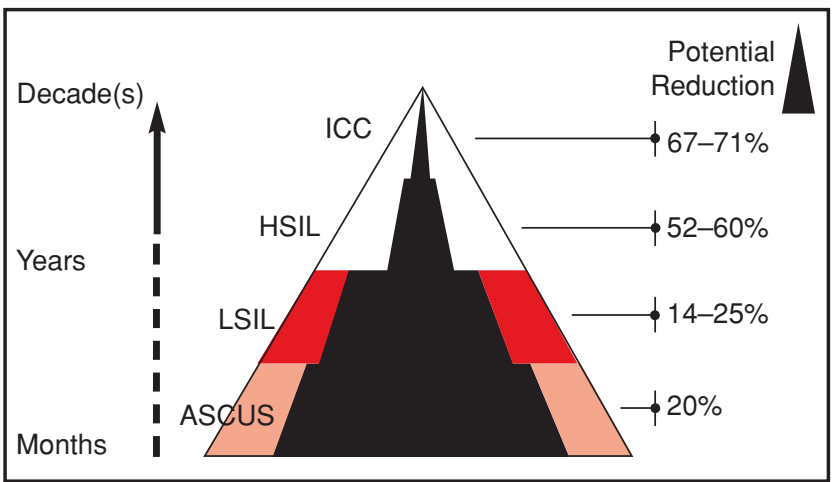

Figure 2 Predicted impact of a vaccine including human papillomavirus (HPV) types 16 and 18. ASCUS, atypical squamous cells of undetermined significance; HSIL, high-grade squamous intraepithelial lesion; ICC, invasive cervical carcinoma; LSIL, lowgrade squamous intraepithelial lesion 
and adenocarcinoma in situ. This reduction in cytological abnormalities, treatment of CIN and its associated followup is likely to have the greatest impact in the UK and other developed countries in terms of health care costs and the social and emotional costs to women. After 1-3 decades the vaccinated cohort should show a reduction of $70 \%$ in the incidence of cervix cancer and if screening has continued and if its coverage has been maintained at current levels then the reduction could reach more than 90\%. If HPV 6 and 11 are included in the vaccine mix then in a reasonably short time period genital wart incidence would be reduced by more than $90 \%$. This really does look like the beginning of the end for HPV-associated disease in women.

Statements on funding and competing interests

Funding None identified.

Competing interests The author is a consultant for Merck Vaccines, GlaxoSmithKline Biologicals and Sanofi Pasteur MSD.

References

1 Parkin DM, Bray F. Chapter 2: The burden of HPV-related cancers. Vaccine 2006; 24(Suppl. 3): S11-S25.

2 Garland SM, Hernandez-Avila M, Wheeler CM, et al. Quadrivalent vaccine against human papillomavirus to prevent anogenital diseases. N Engl J Med. 2007; 356: 1928-1943.

3 Ault KA. Effect of prophylactic human papillomavirus L1 viruslike-particle vaccine on risk of cervical intraepithelial neoplasia grade 2 , grade 3 , and adenocarcinoma in situ: a combined analysis of four randomised clinical trials. Lancet 2007; 369: 1861-1868.

4 Paavonen J, Jenkins D, Bosch FX, Naud P, Salmerón J, Wheeler CM, et al.; HPV PATRICIA study group. Efficacy of a prophylactic adjuvanted bivalent L1 virus-like-particle vaccine against infection with human papillomavirus types 16 and 18 in young women: an interim analysis of a phase III double-blind, randomised controlled trial. Lancet 2007; 369: 2161-2170.

5 Centres for Disease Control and Prevention (CDC). http://www.cdc.gov/nip/ACIP/slides/feb07/08-hpv-2-barr.pdf [Accessed 5 August 2007]

6 Fraser C, Tomassini JE, Xi L, Golm G, Watson M, Giuliano AR, et al. Modeling the long-term antibody response of a human papillomavirus (HPV) virus-like particle (VLP) type 16 prophylactic vaccine Vaccine 2007: 25: 4324-4333.

7 Olsson SE, Villa LL, Costa RL, Petta CA, Andrade RP, Malm C, et al. Induction of immune memory following administration of a prophylactic quadrivalent human papillomavirus (HPV) types 6/11/16/18 L1 virus-like particle (VLP) vaccine. Vaccine 2007; 25: 4931-4939.

8 Giannini SL, Hanon E, Moris P, Van Mechelen M, Morel S, Dessy $F$, et al. Enhanced humoral and memory B cellular immunity using HPV16/18 L1 VLP vaccine formulated with the MPL/aluminium salt combination (AS04) compared to aluminium salt only. Vaccine 2006; 24: 5937-5949.

9 Block SL, Nolan T, Sattler C, Barr E, Giacoletti KE, Marchant CD, et al.; Protocol 016 Study Group. Comparison of the immunogenicity and reactogenicity of a prophylactic quadrivalent human papillomavirus (types $6,11,16$, and 18) $L 1$ virus-like particle vaccine in male and female adolescents and young adult women. Pediatrics 2006; 118: 2135-2145.

10 Bosch FX, de Sanjosé S. Chapter 1: Human papillomavirus and cervical cancer - burden and assessment of causality. J Natl Cancer Inst Monogr 2003; 31: 3-13.

11 Sasieni P, Adams J, Cuzick J. Benefits of cervical screening at different ages: evidence from the UK audit of screening histories. Br J Cancer 2003; 89: 88-93.

12 Kitchener HC, Castle PE, Cox JT. Chapter 7: Achievements and limitations of cervical cytology screening. Vaccine 2006; 24(Suppl. 3): S63-S70.

13 Clifford G, Franceschi S, Diaz M, Muñoz N, Villa LL. Chapter 3: HPV type-distribution in women with and without cervical neoplastic diseases. Vaccine 2006; 24(Suppl. 3): S26-S34.

\section{FACULTY MEMBERSHIP EXAMINATION}

The Membership Examination (MFFP) consists of:

\section{Part 1 Multiple Choice Question Paper (MCQ)}

This 11/2-hour paper consists of 50 clinical science and applied science questions.

The examination will be held in London on Wednesday 23 April 2008 and Friday 17 October 2008. Applications for April 2008 must be received by 1 January 2008 and those for October 2008 must be received by 1 July 2008. The application form and information on the Part 1 can be obtained from the Faculty of Family Planning website (www.ffprhc.org.uk).

\section{$\square$ Dissertation or Case Reports}

Submission of one Dissertation (10 000 words) or two Case Reports (3000 words each).

Please visit the Faculty of Family Planning website (www.ffprhc.org.uk) for the latest changes to this part of the examination, and for information on exemptions.

\section{$\square$ Part 2 Examination (CRQ, MEQ, OSCE)}

This all day examination consists of:

Critical Reading Question examination paper (CRQ)

Modified Essay Question examination paper (MEQ)

Objective Structured Clinical Examination (OSCE)

Applications for the MFFP Part 2 held in June 2008 must be received by 3 January 2008. Information on the Part 2 examination, the Examination Regulations and the application form appear on the Faculty of Family Planning website (www.ffprhc.org.uk).

The qualification is subject to re-certification every 5 years.

For the MFFP Examination Regulations (December 2005), information and application forms please visit the Faculty of Family Planning website: www.ffprhc.org.uk (see Training \& Exams and MFFP Member). Also available on request from: Mrs Denise Pickford, Examinations, Faculty of Family Planning and Reproductive Health Care of the Royal College of Obstetricians and Gynaecologists, 27 Sussex Place, Regent's Park, London NW1 4RG, UK. Tel: +44 (0) 207724 5629. Fax: +44 (0) 20 7723 5333. E-mail: denise@ffprhc.org.uk 